\title{
Polarization control of a free-electron laser oscillator using helical undulators of opposite helicities
}

\author{
Jun Yan $\odot,{ }^{1,2, *}$ Hao Hao, ${ }^{1,2}$ Senlin Huang $\odot{ }^{3}$ Jingyi Li, ${ }^{4}$ Vladimir N. Litvinenko $\odot,{ }^{5}$ \\ Peifan Liu $\odot,{ }^{1,2}$ Stepan F. Mikhailov, ${ }^{1,2}$ Victor G. Popov $\oplus^{1,2}$ Gary Swift, ${ }^{1,2}$ \\ Nikolay A. Vinokurov $\odot^{6,7}$ and Ying K. Wu $\odot^{1,2, \uparrow}$ \\ ${ }^{1}$ Department of Physics, Duke University, Durham, North Carolina 27708, USA \\ ${ }^{2}$ Triangle Universities Nuclear Laboratory, Durham, North Carolina 27708, USA \\ ${ }^{3}$ Institute of Heavy Ion Physics, School of Physics, Peking University, Beijing 100871, China \\ ${ }^{4}$ Institute of High Energy Physics, Chinese Academy of Sciences, Beijing 100049, China \\ ${ }^{5}$ Department of Physics and Astronomy, Stony Brook University, Stony Brook, New York 11794, USA \\ ${ }^{6}$ Budker Institute of Nuclear Physics SB RAS, Novosibirsk 630090, Russia \\ ${ }^{7}$ Novosibirsk State University, Novosibirsk 630090, Russia
}

(Received 8 April 2020; accepted 18 June 2020; published 29 June 2020)

\begin{abstract}
Polarized photon beams provide a unique experimental tool for the study of various polarizationdependent physical processes. Here, we report the experimental demonstration of full polarization control of an oscillator free-electron laser (FEL) using helical undulators of opposite helicities. Using two helical undulator magnets of opposite helicities and a buncher magnet in between, we have generated a linearly polarized FEL beam with any desirable polarization direction. With the development of a high-precision FEL polarimeter, we are able to optimize the highly polarized FEL beams in visible wavelengths and measure the polarization with high accuracy, demonstrating linear polarization $P_{\operatorname{lin}}>0.99$ on the routine basis and with the maximum polarization reaching $P_{\text {lin }}=0.998$. In this paper, we describe the FEL configuration, experimental setup, and related beam diagnostics, including the newly developed highprecision FEL polarimeter. We report our experimental approaches to generate, tune up, and characterize the polarization controllable FEL beams and share a new insight into how high-degree polarization is realized based upon our investigation of the temporal structure of the FEL beam. This FEL polarization control technique has been used successfully to generate a polarization controllable Compton $\gamma$-ray beam for nuclear physics experiments.
\end{abstract}

DOI: 10.1103/PhysRevAccelBeams.23.060702

\section{INTRODUCTION}

The free-electron laser (FEL), driven by either a storage ring or a linac, is widely used to produce wavelengthtunable, high-intensity coherent radiation [1-5]. The invention of the FEL has opened up a wide range of opportunities for experimental research in condensed matter physics, chemistry, material sciences, and biomedical sciences. With a number of FELs constructed and commissioned to deliver high-quality laser beams for user experiments in the past few decades [6], the FEL community has increasingly expanded its exploration of novel FEL capabilities beyond the wavelength tunability [7-15]. In particular, polarization

\footnotetext{
*junyan@fel.duke.edu

wu@fel.duke.edu
}

Published by the American Physical Society under the terms of the Creative Commons Attribution 4.0 International license. Further distribution of this work must maintain attribution to the author(s) and the published article's title, journal citation, and DOI. control [16-19] has been extensively investigated for several types of FELs. Photon beam polarization is a unique experimental tool for studying various polarization-dependent physical, chemical, and biological phenomena. For example, to produce high-resolution images of certain biological tissues, some biomedical imaging techniques utilize the polarization-based contrast mechanism by switching the polarization of the light sources used in imaging [20,21]. Using a photon beam with controllable polarization, linear or circular dichroism techniques can be used to characterize protein structures in the visible and ultraviolet regions $[22,23]$ or to investigate the magnetic and electronic properties of various magnetic materials in the $\mathrm{x}$-ray regime [24,25]. In addition, when a polarization tunable FEL is used to drive a Compton scattering $\gamma$-ray source, the resulting $\gamma$-ray beam with variable polarization can serve as a powerful probe to explore nucleon structures by measuring the related spin polarizabilities [26-31].

Polarization of light can be controlled in different ways for different spectral regions. In the visible regime, polarization can be manipulated using conventional polarizing 
optics via photon-matter interactions. For example, a linear polarizer is typically used to polarize the incident light by constraining in a particular direction the oscillation of bound electrons inside the polarizer material and, therefore, the direction of the reemitted electric field. Optical wave plates are also commonly employed to vary the polarization states of the incoming waves by inducing an extra phase delay to one of the two electric field components. In shorter wavelength regions (e.g., beyond ultraviolet), polarizing optics either are not available or have very limited capabilities. In this region, polarization of light is mainly determined by its production mechanism. For the accelerator-based light sources using relativistic electrons as the radiation source, photon beam polarization can be manipulated via controlling the motions of electrons in some specially arranged static magnetic fields or electromagnetic fields. One wellestablished polarization control technique for synchrotron radiation sources and FELs employs elliptically polarized undulators with movable magnet arrays [19,32]. A specific polarization state can be obtained by mechanically moving one or more magnet arrays to form a specific magnetic configuration. However, compared to the conventional planar and helical undulators, the manufacturing of such an undulator is more complex and costly.

An alternative polarization control method is to utilize two sets of orthogonally polarized undulators with a phase shift magnet placed in between. With such a crossed undulator configuration, photon beam polarization can be controlled by properly mixing two orthogonal beam components with a variable phase shift. This idea was first proposed in the 1980s [33] for direct spontaneous radiation using undulators and experimentally explored at the storage ring BESSY in the early 1990s by using two crossed planar undulators and a monochromator to produce circularly polarized radiation [34]. Later on, polarization control using crossed planar undulators was proposed for an FEL [35]. In 2005, polarization control of an FEL was first experimentally demonstrated at the Duke storage ring FEL facility using two planar and two helical undulators [36]. At that time, the polarization states were limited to horizontally linear and elliptical polarizations, due to a nonoptimal set of undulators available. Since then, the crossed undulator configuration has been theoretically explored [37-41] and experimentally studied using a few single-pass FELs $[17,18,42]$. However, the degree of polarization which can be attained in these systems is relatively low for a few reasons. In a self-amplified spontaneous emission FEL, the head of the optical pulse or wave packet does not interact well with the tail of the pulse, resulting in a relatively low longitudinal coherence and, therefore, a low degree of desirable polarization [37]. In a seeded FEL with much improved longitudinal coherence, the low degree of polarization is mainly attributed to either an imperfect transverse superposition or an optical phase mismatch of the radiation from the two different undulators along the electron beam path $[17,18]$. In contrast, in an FEL oscillator the FEL slippage plays a critical role in building up the coherent state of polarization along the optical wave packet as the FEL interaction is repeated in many passes with the wave packet circulating in the cavity. In addition, the shared optical cavity of an FEL oscillator ensures the identical transverse beam properties for the two cavity modes with the same frequency but different polarization states. These advantages make the FEL oscillator well suited for producing photon beams with the purest polarization.

With the upgrades of various critical components of the Duke FEL system as well as the development of a highprecision polarization diagnostic system, a dedicated FEL polarimeter, we have developed a new mode of the FEL oscillator operation with full polarization control for the first time. This new operational mode allows us to readily switch among the left- and right-circular polarizations and linear polarization at any desirable polarization direction. In particular, a linearly polarized FEL beam has been produced with a rotatable polarization direction and an unprecedentedly high degree of polarization. Such an FEL beam has been successfully utilized to generate a polarization switchable $\gamma$-ray beam for nuclear physics research at the High Intensity Gamma-ray Source (HIGS), as reported in a recent publication [43]. In contrast to Ref. [43], which describes $\gamma$-ray polarization control with very limited information on the FEL subsystem itself, here we provide a more extensive description of our visiblerange variable-polarization FEL with details of potential interest to FEL physicists. In this paper, we will provide a detailed description of our experimental setup and diagnostics, including the FEL configuration and the FEL polarimeter. In addition, our experimental approaches to generate, tune up, and characterize the polarization controllable FEL beams will be reported. Finally, we will share a new insight into how high-degree polarization is realized based upon our investigation of the temporal structure of the FEL beam.

\section{MACHINE LAYOUT}

The Duke FEL is an oscillator driven by a storage ring. The storage ring with a circumference of $107.46 \mathrm{~m}$ can be operated from $240 \mathrm{MeV}$ up to $1.2 \mathrm{GeV}$. The FEL system is comprised of an optical cavity and an undulator system, located in one of the storage ring straight sections, as shown in Fig. 1. The near-concentric FEL optical cavity is formed by two high-reflectivity mirrors with a cavity length of $53.73 \mathrm{~m}$, and the round-trip time of the trapped optical beam is matched with the electron beam revolution time in the storage ring. The undulator system allows the simultaneous use of up to four helical OK-5 undulators, and the FEL can be configured to use any subset of undulators [44].

The FEL mainly serves the HIGS, a polarized Compton backscattering $\gamma$-ray source $[45,46]$. With its wavelength 


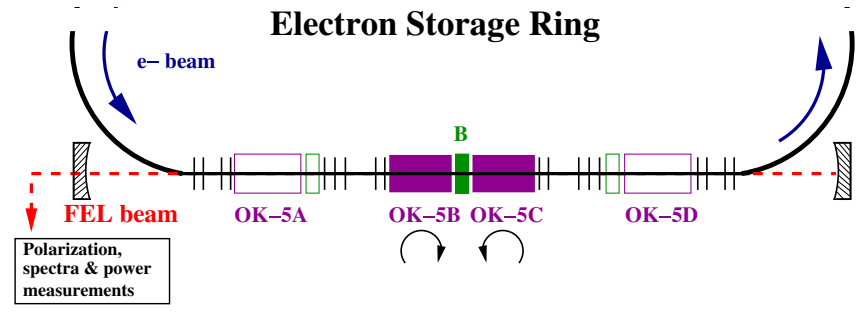

FIG. 1. Undulator configuration for the polarization control of FEL beams. The two helical undulators in the middle of the FEL straight section (OK-5B and $\mathrm{OK}-5 \mathrm{C})$ are turned on and operated in opposite helicities. Buncher B is used as a phase shifter.

tunable from 1060 down to $190 \mathrm{~nm}$, this FEL not only supports the production of $\gamma$-ray beams in a wide energy range $(1-100 \mathrm{MeV})$, but also allows for the generation of high-flux $\gamma$-ray beams by colliding the electron beam with the laser beam inside the optical cavity. To incorporate polarization control into this FEL, it is imperative to develop an optical beam manipulation technique without using conventional polarizing optics. This is because these optics, if installed inside the FEL cavity, will limit the attainable intracavity power due to thermal loading and radiation damage and, thus, degrade the FEL performance.

Our solution is to use helical undulators of opposite helicities for optics-free polarization control of the FEL beam. In contrast to the original scheme proposed with crossed planar undulators [35], using helical ones not only allows for more efficient coupling between the electron and FEL beams, but also significantly reduces the thermal loading on the downstream FEL mirror by taking advantage of the natural off-axis distribution of high-order harmonic radiation from helical undulators. In this work, we have achieved full control of FEL beam polarization using either two (OK-5B and OK-5C) or all four helical undulators (see Fig. 1). While the two upstream undulators (OK-5A and OK-5B) are wired up to have a fixed helicity, the two downstream ones (OK-5C and OK-5D) are configured with switchable helicity by using a solid-state switch to reverse the direction of the dc current only in the vertical undulator coil. In this paper, we report the experimental results using the two-undulator configuration, where OK-5B and OK-5C are powered independently with opposite helicities (see Fig. 1). A buncher magnet (denoted as B in Fig. 1) is installed between OK-5B and OK-5C undulators, so that these three magnets form an optical klystron [47]. With two helical undulators set to have (almost) the same current, a linearly polarized FEL beam can be produced with careful tuning of the system. This beam may be considered as a superposition of a set of FEL cavity modes (with slightly different frequencies); each of them is a linear combination of two degenerate eigenmodes with different polarizations, for example, a linear combination of vertically and horizontally polarized eigenmodes. The optical klystron magnetic system is tuned to obtain the maximum gain for the required polarization, and, in our case, it is linear polarization with a given direction.

\section{FEL POLARIZATION DIAGNOSTICS}

In this work, high-quality FEL polarization diagnostics have been developed in multiple stages with increasing accuracy. The polarization of the FEL beam is characterized using the normalized Stokes vector (a column vector): $\vec{S}=\left(1, S_{1}, S_{2}, S_{3}\right)^{T}$, where $S_{1}$ or $S_{2}$ represents the relative intensity asymmetry of the horizontal and vertical polarization components or that of $45^{\circ}$ and $135^{\circ}$ linear polarization components, respectively, and $S_{3}$ represents the relative intensity asymmetry of the right- and left-circular polarization components. We measure the polarization of the FEL beam after it is extracted through an optically isotropic $\mathrm{CaF}_{2}$ exit window.

We first developed a prototype FEL polarimeter, as shown in Fig. 2(a). This polarization diagnostic system starts from
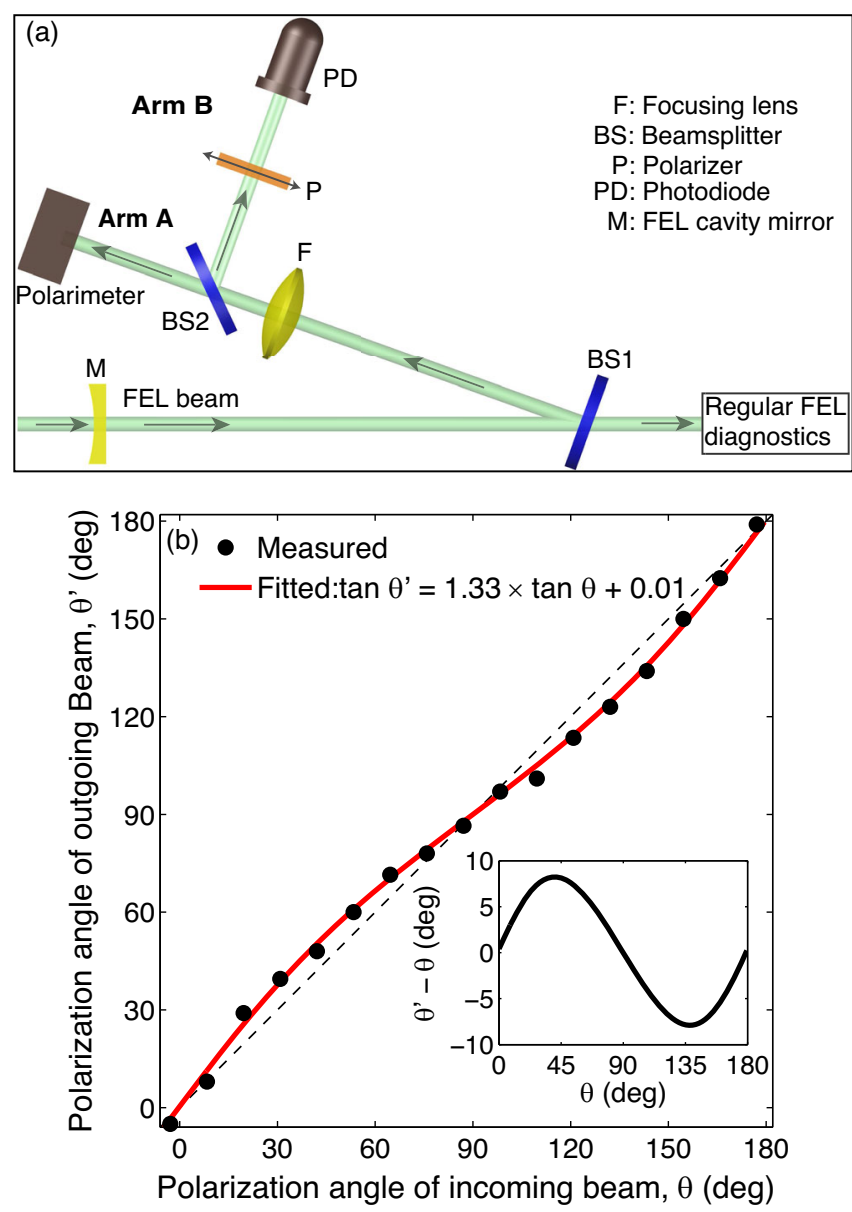

FIG. 2. (a) Layout of the prototype FEL polarimeter with two nonpolarizing beam splitters (BS1 and BS2), a focusing lens (F), a linear polarizer $(\mathrm{P})$, a commercial polarimeter, and a photodiode (PD). (b) Polarization angle measured in arm B as a function of the polarization angle of an incoming linearly polarized beam at $548 \mathrm{~nm}$. 
beam splitter BS1 and utilizes the beam reflected off BS1 at a small angle while the transmitted beam is sent to the existing regular FEL diagnostics, which measure the beam profile, spectrum, power, etc. Inside this prototype polarimeter, after a focusing lens $(\mathrm{F})$, the beam is split a second time using beam splitter BS2. In the transmission arm $(\operatorname{arm} \mathrm{A})$, the beam is sent into a commercial polarimeter (Thorlabs PA510), a device which measures Stokes parameters using a rotating quarter-wave plate. This commercial polarimeter is used for real-time monitoring of overall FEL beam polarization with limited capabilities. In the reflection arm of BS2 (arm B), a dedicated optical setup is used to precisely measure the linear polarization of the FEL beam using a manually rotatable polarizer $(\mathrm{P})$ and a photodiode (PD). The linear polarization state of the incoming beam can be obtained by measuring the maximum intensity $\left(I_{\max }\right)$ and minimum intensity $\left(I_{\min }\right)$ while rotating polarizer P. This measurement determines both the total degree of linear polarization of the beam, as described by the beam's intensity asymmetry $\mathcal{A}=\left(I_{\max }-I_{\min }\right) /\left(I_{\max }+I_{\min }\right)$, and the polarization direction, which is recorded using the angle $\theta$ between the major axis of the polarization ellipse and the horizontal direction (the $+x$ direction in our coordinate system).

Any real optical element with nonideal transmission properties can adversely impact the polarization measurement. Therefore, we adopted two strategies to minimize such effects. First, we devised a setup with a minimum number of optically nonactive (i.e., birefringence-free) elements for linear polarization measurement. Second, we devoted substantial effort to studying the impact of various optical components - this led to the development of an effective, yet straightforward calibration method which significantly improved the measurement accuracy. This method is used to compensate for the most significant error term, an unequal transmission of the electric field in two mutually perpendicular directions for a system using lowbirefringence optics. In our setup [Fig. 2(a)], the most important error sources are the two beam splitters. It is also worth pointing out that the calibration of the polarimeter depends on the optical wavelength and should be carried out for each wavelength of the FEL operation. In this paper, we report only the calibration results of the prototype polarimeter (and later that of a production polarimeter) for an FEL beam around $548 \mathrm{~nm}$.

Let us consider the effect of the laser beam electric field after being reflected or transmitted by an optical element:

$$
\begin{aligned}
& E_{x}^{\prime}=p_{x} E_{x}, \\
& E_{y}^{\prime}=p_{y} E_{y},
\end{aligned}
$$

where $E_{x}$ and $E_{y}$ are the horizontal and vertical electric fields of the incident beam, respectively, and the coefficients $p_{x}$ and $p_{y}$ describe the change of the electric fields in the horizontal ( $x$ direction) and vertical ( $y$ direction) directions, respectively, when the light is reflected from or transmitted through the optical element. When $p_{x} \neq p_{y}$, this element will change the incoming light's polarization. It should be noted that $p_{x}$ and $p_{y}$ for a generic optical element are complex numbers, which account for both amplitude and phase changes of the light's horizontal and vertical components. However, in our case, because we deliberately choose extremely low-birefringence materials for transporting the FEL beams, $p_{x}$ and $p_{y}$ are considered to be real numbers, describing the amplitude attenuation. For a beam splitter, the deviation of the ratio $r=p_{y} / p_{x}$ from unity varies with the incident angle of the beam and is minimum for angles close to normal incidence. In this setup [Fig. 2(a)], the most important contributions come from two beam splitters BS1 and BS2, especially BS2 with a large incident angle. The effect of BS1 is reduced by choosing a small reflection angle of about $7.5^{\circ}$ as permitted by available space on the optical table.

The polarization angle $\theta$ of a linearly polarized light beam is given by $\tan \theta=E_{y} / E_{x}$. It is worth noting that, for a general elliptically polarized beam, this angle would indicate the direction of the major axis of the associated polarization ellipse. Using Eqs. (1), the polarization angle $\theta^{\prime}$ of the outgoing beam after BS2 and the polarization angle $\theta$ of the incoming beam before BS1 are related by

$$
\tan \theta^{\prime}=r \tan \theta
$$

This relationship allows us to experimentally determine $r$, the ratio of the overall transmission for the beam going through both beam splitters. The source beam used for the measurement of $r$ was prepared by passing a circularly polarized FEL beam through a rotatable linear polarizer with its polarization axis set at angle $\theta$ relative to the $x$ direction. The polarization direction of the outgoing beam, $\theta^{\prime}$, was then measured in arm B by rotating polarizer P. The relationship between $\theta$ and $\theta^{\prime}$ was fitted using Eq. (2), as shown in Fig. 2(b). Ratio $r$ is shown as the slope of the linear fit, which is found to be $1.326 \pm 0.004$. Because of this unequal transmission in the horizontal and vertical directions, the change of the polarization direction is maximum $\left(\Delta \theta=\theta^{\prime}-\theta \sim 8^{\circ}\right)$ when the incoming polarization angle $\theta$ is close to $45^{\circ}$ (or $135^{\circ}$ ) and minimum when the incoming beam is horizontally or vertically polarized. Knowing the ratio $r$, the polarization angle of the incoming FEL beam can be obtained by measuring that of the outgoing beam and applying Eq. (2).

The knowledge of the transmission ratio $r$ also allows us to perform corrections on the measured degree of linear polarization. Assuming a fully polarized beam, the intensity asymmetry $\mathcal{A}$ measured in arm B gives the normalized Stokes vector $\vec{S}^{\prime}$ of the outgoing FEL beam, which can then be used to retrieve $\vec{S}$ of the incoming FEL beam using the relationship [48] 


$$
\vec{S}=\frac{I_{0}^{\prime}}{I_{0}} M_{\mathrm{AT}}^{-1}\left(p_{x}, r\right) M_{\mathrm{R}}^{-1}\left(\theta^{\prime}\right) \vec{S}^{\prime}(\mathcal{A}),
$$

where $I_{0}$ and $I_{0}^{\prime}$ are the intensity of the incoming and outgoing beams, respectively. In this equation,

$$
M_{\mathrm{AT}}\left(p_{x}, r\right)=\frac{p_{x}^{2}}{2}\left(\begin{array}{cccc}
1+r^{2} & 1-r^{2} & 0 & 0 \\
1-r^{2} & 1+r^{2} & 0 & 0 \\
0 & 0 & 2 r & 0 \\
0 & 0 & 0 & 2 r
\end{array}\right)
$$

is the Mueller matrix for the transmission ratio of the horizontal and vertical electric fields with $r=p_{y} / p_{x}$, and

$$
M_{\mathrm{R}}\left(\theta^{\prime}\right)=\left(\begin{array}{cccc}
1 & 0 & 0 & 0 \\
0 & \cos \left(2 \theta^{\prime}\right) & \sin \left(2 \theta^{\prime}\right) & 0 \\
0 & -\sin \left(2 \theta^{\prime}\right) & \cos \left(2 \theta^{\prime}\right) & 0 \\
0 & 0 & 0 & 1
\end{array}\right)
$$

is the Mueller matrix representing the rotation of the coordinate system by angle $\theta^{\prime}$. This angle describes the direction of the linear polarization as measured by polarizer $\mathrm{P}$ after the beam has been transported by optics in the polarimeter. Upon obtaining the normalized Stokes vector $\vec{S}$, using Eq. (3) the degree of linear polarization of the incoming FEL beam can then be calculated:

$$
P_{\text {lin }}=\sqrt{S_{1}^{2}+S_{2}^{2}} .
$$

Using a calibrated prototype FEL polarimeter, the highest degree of linear polarization obtained for the FEL operation using helical undulators of opposite helicities was about $P_{\text {lin }}=0.96$, a very good result already. However, further investigation revealed that the FEL operation was not fully optimized to achieve even purer linear polarization. This was because the FEL tuning was carried out while monitoring the polarization change in real time using the commercial polarimeter in arm A. However, proper operation of this commercial instrument requires an incident light beam with stable intensity due to the use of a rotating quarter-wave plate inside. The intrinsic power fluctuations of our FEL beam at a timescale comparable to the rotation period of the quarter-wave plate (tens of hertz) can significantly degrade the measurement accuracy. Despite our best effort to keep the FEL beam intensity stable while performing tuning for linear polarization, this commercial device fundamentally limited our ability to further optimize the FEL operation. It is also worth mentioning the other limitation of this device-it has a limited workable wavelength in the visible range from 450 to $700 \mathrm{~nm}$, narrower than our intended wider wavelength coverage of the already deployed conventional FEL diagnostic optics.
To reliably measure FEL beam polarization with improved accuracy over a wider wavelength range from near infrared $(1060 \mathrm{~nm})$ to ultraviolet $(350 \mathrm{~nm})$, we have developed a new polarization diagnostic system, an FEL polarimeter for routine operation. This uses a set of nonpolarizing optics with negligible birefringence and highquality polarizing optical components, including Glan Thompson beam splitters with a high extinction ratio [49]. Furthermore, no rotating component is used in the system with all optical components fixed in space during measurements. A full characterization of the beam polarization requires simultaneous measurements of all three Stokes components $S_{1}, S_{2}$, and $S_{3}$. Under a reasonable assumption that the FEL beam is completely polarized, a simpler setup to measure only two Stokes parameters is possible. Our FEL polarimeter as shown in Fig. 3(a) is comprised of two arms, each of which can measure the intensities of two orthogonal linear polarization components using a Glan Thompson beam splitter (manually rotatable) and two photodiodes. To work with a light beam mostly in linear polarization, this system can be configured in two modes-Mode one (the real-time monitor mode): Arm A and arm B simultaneously measure two independent sets of intensity asymmetry (Stokes parameters $S_{1}$ and $S_{2}$ ) without moving or rotating any optical elements so that a polarization ellipse can be constructed in real time; mode two (precision mode for linear polarization): Only one of the arms (usually arm A) is used to carry out an asymmetry measurement to precisely determine the degree of linear polarization by manually rotating the corresponding Glan Thompson beam splitter. Such a measurement is similar to the measurement performed using arm B of the prototype system [Fig. 2(a)]. Mode one with fixed optics is useful for the tuning and optimization of the FEL beam polarization. However, compared to mode two, the measurement accuracy of the linear polarization in mode one is somewhat lower, because four intensity measurements in two different arms need to be accurately cross-calibrated. Therefore, after tuning up the FEL, accurate polarization measurements are carried out using mode two.

The same transmission calibration technique developed for the prototype system has been carried out for this production system, which gives a transmission ratio $r_{A}=$ $0.815 \pm 0.005$ for arm $\mathrm{A}$ and $r_{B}=1.186 \pm 0.007$ for arm B. Applying these corrections for both arms A and $\mathrm{B}$, the accuracy of this FEL polarimeter for measuring a linearly polarized incident beam has been evaluated using the measurement results shown in Fig. 3(b). The incoming linearly polarized beam is prepared by passing an FEL beam through a high-quality linear polarizer with an extinction ratio of $7 \times 10^{-5}$ (at wavelength $548 \mathrm{~nm}$ ) and expected degree of linear polarization 0.9999 . The polarization angle of this beam with almost perfect linear polarization is then varied by rotating the linear polarizer; at each angle, the outgoing beam polarization is measured 

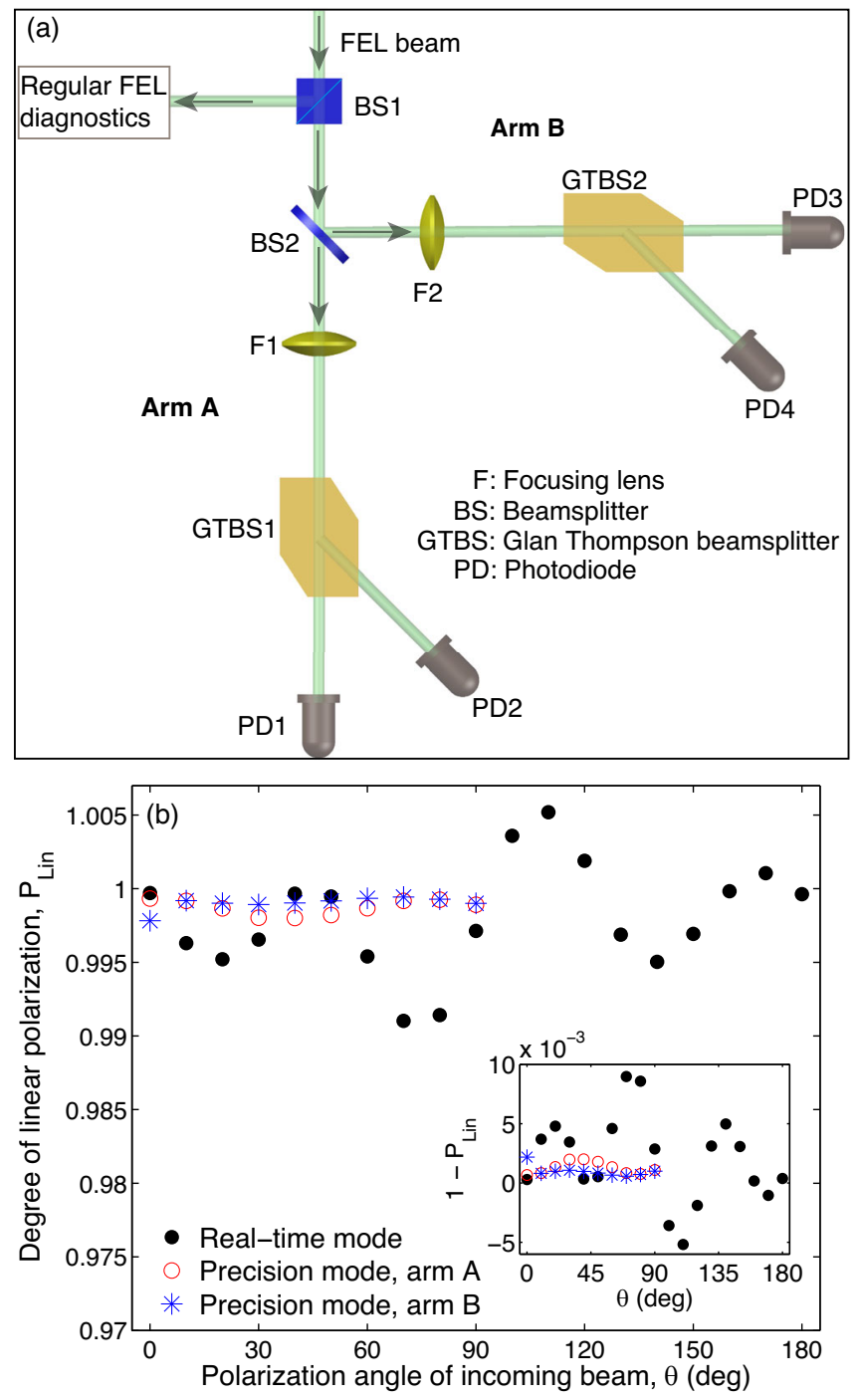

FIG. 3. (a) Layout of the new FEL polarimeter. After the FEL beam passes through a nonpolarizing beam splitter (BS1), it is split into two arms by a second nonpolarizing beam splitter (BS2), each comprised of a focusing lens, a Glan Thompson beam splitter, and two photodiodes. (b) Measured degree of linear polarization $P_{\text {lin }}$ in different operational modes when the incoming beam has nearly perfect linear polarization as it is prepared using a high-quality linear polarizer. The inset shows the deviation from a perfect linear polarization, $\Delta P_{\text {lin }}=1-P_{\text {lin }}$.

using our system. In the real-time mode with fixed beam splitters, the measured deviation from perfect linear polarization $\Delta P_{\text {lin }}=\left(1-P_{\text {lin }}\right)$ has an average value of $2 \times 10^{-3}$ and an rms variation of $4 \times 10^{-3}$ as the polarization angle is varied between 0 and $180^{\circ}$. In the real-time mode, the $P_{\text {lin }}$ values at a few data points exceed unity slightly (up to a few $10^{-3}$ ); this is caused by small calibration errors in the beam intensity measurements between the two arms. In the precision mode with the Glan Thompson beam splitter manually rotated to follow the polarization direction (i.e., the direction with maximum intensity), $\Delta P_{\text {lin }}$ is found to have an average value of $1 \times 10^{-3}$ with an rms variation of
$5 \times 10^{-4}$ for both arms as the polarization angle is varied between 0 and $90^{\circ}$, showing more consistent results. Based upon these results, we determine that the overall accuracy of this FEL polarimeter with careful calibration is about a few $10^{-3}$ in the real-time mode and $10^{-3}$ in the precision mode when used to measure $P_{\text {lin }}$ for a completely polarized beam with substantial linear polarization. It is worth pointing out that, while the above performance of this production polarimeter is determined for $548 \mathrm{~nm}$, consistently good performance can be expected for the entire operation range $(350-1060 \mathrm{~nm})$ if the system is properly calibrated for each operational wavelength.

\section{POLARIZATION CONTROL AND CHARACTERIZATION}

To generate a linearly polarized FEL beam using cavity eigenmodes of different polarization states, it is necessary to tune up the system to ensure that any paired eigenmodes with the same frequency but opposite handedness are fully degenerate. With a shared optical cavity and a small singlepass gain, the FEL oscillator is capable of supporting these paired eigenmodes with excellent degeneracy and therefore well-matched gains to build up in multiple passes to reach saturation. From the FEL physics point of view, such precision control of the FEL polarization is achieved by properly choosing the polarization gain matrix elements, which allows the FEL beam to reach saturation with equal intensity for two different circular polarizations.

In terms of physical implementation, two mechanically identical OK-5 undulators, which are nearly symmetrically located around the center of the laser cavity, are employed to produce radiation of opposite circular polarizations with closely matched gains. The FEL gain matching is further improved by aligning the electron beam orbit in both FEL undulators to ensure the consistency of the electron-laser coupling inside the two undulators. In order to achieve the highest degree of linear polarization, careful tuning of various machine settings is required-in addition to the electron beam orbit, other important knobs include the undulator current, buncher setting, alignment of the FEL optical axis, and FEL detuning. In our case, the FEL detuning is realized by changing the storage ring $\mathrm{rf}$ frequency to adjust the revolution time of the electron beam relative to the FEL.

At a particular polarization angle, the FEL is tuned in a few iterations-the tuning knobs are first adjusted to optimize the polarization ellipse measured in real time either by the commercial polarimeter in the prototype diagnostic system [see Fig. 2(a)] or using the real-time mode of the new FEL polarimeter [see Fig. 3(a)]. A finer tuning is then performed to maximize the intensity asymmetry $\mathcal{A}$ measured either in arm B of the prototype system or using the precision mode of the production system. Figure 4 shows 17 FEL beam polarization states measured while the FEL is tuned to rotate the polarization angle. 

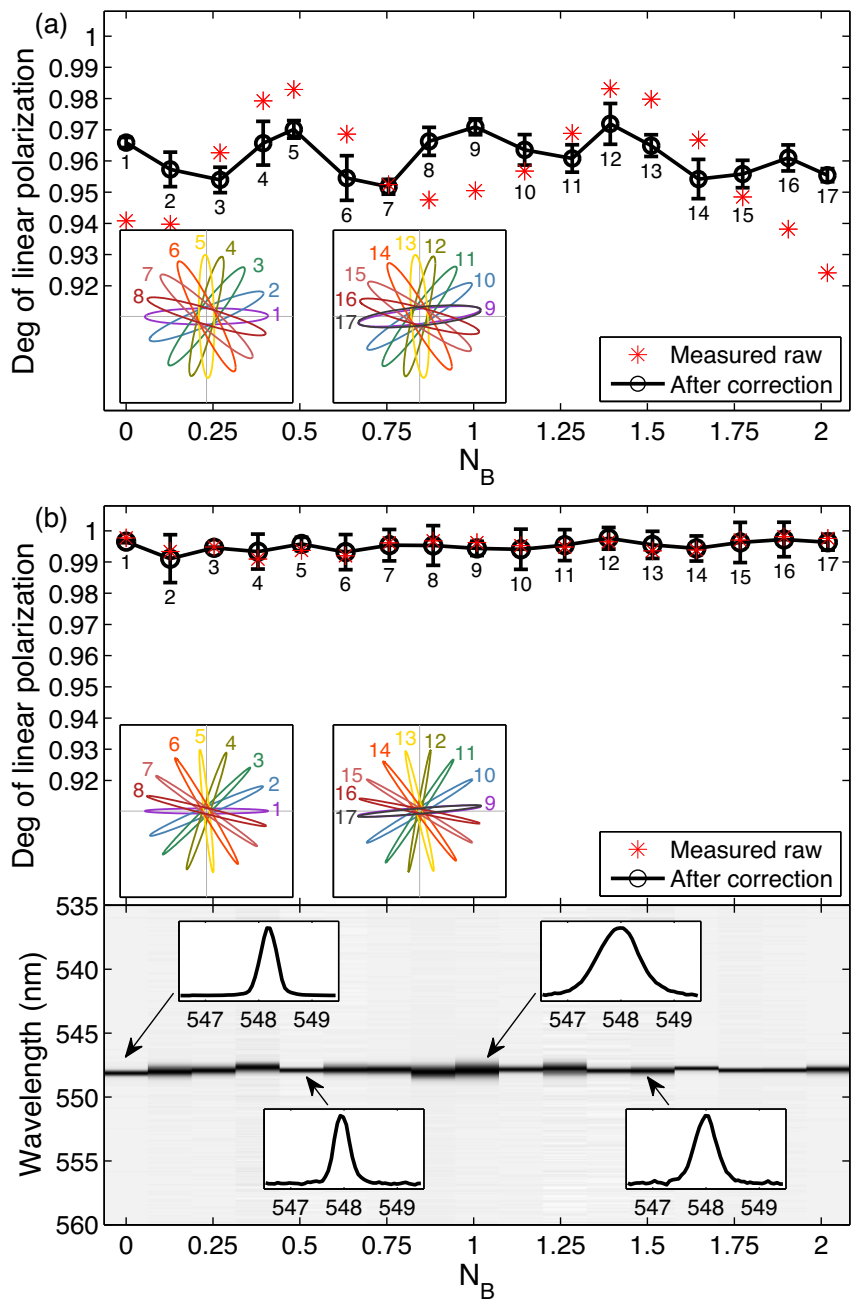

FIG. 4. Degree of FEL beam linear polarization $P_{\text {lin }}$ measured using (a) the prototype and (b) the new FEL polarimeter. The polarization angle is rotated by tuning buncher setting $N_{\mathrm{B}}$. The insets show the measured FEL polarization ellipses. All measurements are indexed by a number from 1 to 17 . In (a), the storage ring is operated with a single-bunch electron beam at an energy of $550 \mathrm{MeV}$ and beam current of about $45 \mathrm{~mA}$. The FEL is lasing at about $547.8 \mathrm{~nm}$ with an FEL detuning of about $7 \times 10^{-7}$. In (b), a $533 \mathrm{MeV}$ single-bunch electron beam is used with a beam current of about $30 \mathrm{~mA}$. The FEL is operated with zero detuning. The lower part of (b) shows the measured spectra of the FEL beam with their maximum intensity scaled to unity. The measured FEL central wavelength is $547.94 \pm 0.093 \mathrm{~nm}$, and the measured average rms spectral width is $0.25 \mathrm{~nm}$ with an $\mathrm{rms}$ variation of $0.066 \mathrm{~nm}$. Note that the dataset shown in (b) is different from the one reported in Ref. [43].

By varying buncher setting $N_{\mathrm{B}}$ from 0 to 2 , the polarization angle of the FEL beam is rotated by about two cycles $\left(360^{\circ}\right)$. Here, $N_{\mathrm{B}}$ is the phase delay (normalized by the lasing wavelength) between the radiation from two OK-5 undulators of opposite handedness due to the additional electron path length in the buncher.

In the early study using the prototype FEL polarimeter, in order to obtain reasonably reliable polarization measurements using the commercial polarimeter, the FEL had to be operated with a relatively large FEL detuning to reach a certain level of required power stability. For example, with a detuning $\left|\Delta T / T_{0}\right| \approx 7 \times 10^{-7}$, where $T_{0}$ is the electron beam revolution time, the highest degree of linear polarization measured was 0.96 as shown in Fig. 4(a), which is a good, but not excellent result.

With the deployment of the new polarimeter, as well as more experience in tuning the FEL, we finally were able to achieve the expected higher degree of linear polarization by operating the FEL near zero FEL detuning. As shown in Fig. 4(b), the FEL beam linear polarization $P_{\text {lin }}$ can be consistently controlled close to or above 0.99 with an average of 0.995 and a maximum as high as 0.998 . Buncher setting $N_{\mathrm{B}}$ is critical in determining the polarization angle. However, adjusting $N_{\mathrm{B}}$ would cause a slight intensity mismatch between the two circular polarization components and, therefore, degrade the linear polarization. In addition, the tuning of $N_{\mathrm{B}}$ would slightly shift the FEL wavelength and vary the FEL power. In the measurements shown in Fig. 4(b), to compensate for these undesirable effects, the currents of both undulators are slightly adjusted within a range of $0.3 \%$ for OK-5B and $0.4 \%$ for OK-5C. As a result, while maintaining a high degree of linear polarization for all polarization angles, the FEL central wavelength is also kept very stably around $\bar{\lambda}=547.94 \mathrm{~nm}$ with an rms variation of $\sigma_{\lambda}=0.093 \mathrm{~nm}\left(\sigma_{\lambda} / \bar{\lambda}=1.7 \times 10^{-4}\right)$, as shown in the lower half of Fig. 4(b), and the FEL power is maintained with reasonable stability, with a relative variation of $6.4 \%$ (rms).

It is found that the correction applied to the polarization using the measured transmission ratio helps improve the consistency of the results among the measurements at different polarization angles. Such a correction is found to be more important for measurements taken using the prototype polarimeter with typically lower degrees of linear polarization. In Fig. 4(a), the correction reduces the rms variation of $P_{\text {lin }}$ at different polarization angles by a factor of 2.7 for measurements performed using the prototype diagnostics. For the measurements using the new polarimeter, a modest reduction factor of 1.2 is observed for FEL beams which already have almost perfect linear polarization [see Fig. 4(b)].

The uncertainty in $P_{\operatorname{lin}}$ is calculated by propagating the uncertainties in $\mathcal{A}, r$, and $\theta^{\prime}$ based on Eqs. (3)-(6). It is found that, among these three error sources, the contribution from the uncertainty in the measurement of polarization angle $\left(\sigma_{\theta^{\prime}}\right)$ is the dominant term. Let us investigate a simple case with $r=1$, which refers to an "ideal" optical system with an identical transmission for the horizontal and vertical components of the FEL beam. For this case, ignoring the uncertainties in $\mathcal{A}$ and $r$, the uncertainty in $P_{\text {lin }}$ (rms) can be written as

$$
\sigma_{P_{\text {lin }}}=\frac{\sqrt{2} \mathcal{A}^{2}}{P_{\operatorname{lin}}}\left|\sin \left(4 \theta^{\prime}\right)\right| \sigma_{\theta^{\prime}},
$$


where the $\left|\sin \left(4 \theta^{\prime}\right)\right|$ dependency shows that the error contribution to $P_{\text {lin }}$ is minimum (zero) at polarization angles $0^{\circ}, 45^{\circ}, 90^{\circ}$, and $135^{\circ}$ and larger at other angles. This is consistent with the uncertainty variations observed in Fig. 4. This dependency [Eq. (7)] is expected-there are two contributions to the degree of linear polarization, $S_{1}$ (horizontal/vertical, $0^{\circ} / 90^{\circ}$ ) and $S_{2}\left(45^{\circ} / 135^{\circ}\right.$ relative to the positive horizontal direction) - at these special angles, the measured linear polarization is insensitive to the angle error. For other angles, the correction of noticeable intensity difference in the transmission (or reflection) associated with horizontal and vertical electric field components is more important in order to achieve high accuracy in polarization measurement. For the measurements shown in Fig. 4, we measured the transmission ratio for two specific mutually perpendicular directions (the horizontal and vertical). This leads to smaller uncertainties for the four related angles $0^{\circ}, 45^{\circ}, 90^{\circ}$, and $135^{\circ}$. If we had performed additional transmission ratio measurements at other angles (a time-consuming and delicate task), it would also reduce the measurement uncertainty for other related angles.

\section{HIGH-DEGREE POLARIZATION CONFIRMED BY FEL TEMPORAL STRUCTURE}

The puzzle seems to remain as to how this oscillator FEL using helical undulators of opposite helicities can produce linearly polarized beams with such high purity. Because the above measured polarization was based upon dc power measurements using photodiodes with an integration time of $0.3 \mathrm{~s}$, these measurements actually averaged over tens of FEL macropulses which are separated by a few to tens of milliseconds [50]. It was then recognized that the study of the FEL temporal structure, i.e., the evolution of the FEL macropulses, would provide us a unique opportunity to examine the growth of the beam components in opposite circular polarization states. To perform such a measurement, a Babinet-Soleil compensator, which was set up as a quarter-wave plate (QWP), was inserted between the focusing lens F1 and the Glan Thompson beam splitter in arm A of the new FEL polarimeter [see Fig. 3(a)]. This QWP would convert the two circularly polarized components of opposite handedness to two mutually perpendicular linear ones, which were then separated by the Glan Thompson beam splitter and measured respectively using two photodiodes connected to an oscilloscope. In the meantime, arm B was used to monitor the beam polarization state.

The temporal structure measurements were carried out using an FEL beam pretuned to possess a high-degree horizontal polarization (consistent with $P_{\text {lin }}>0.99$ given by dc polarization measurements). Figure 5(a) shows one of the four consecutive time domain measurements, one second each, taken simultaneously using two channels of a digital oscilloscope with a sampling rate of 100000 samples per second. To account for the differences in the
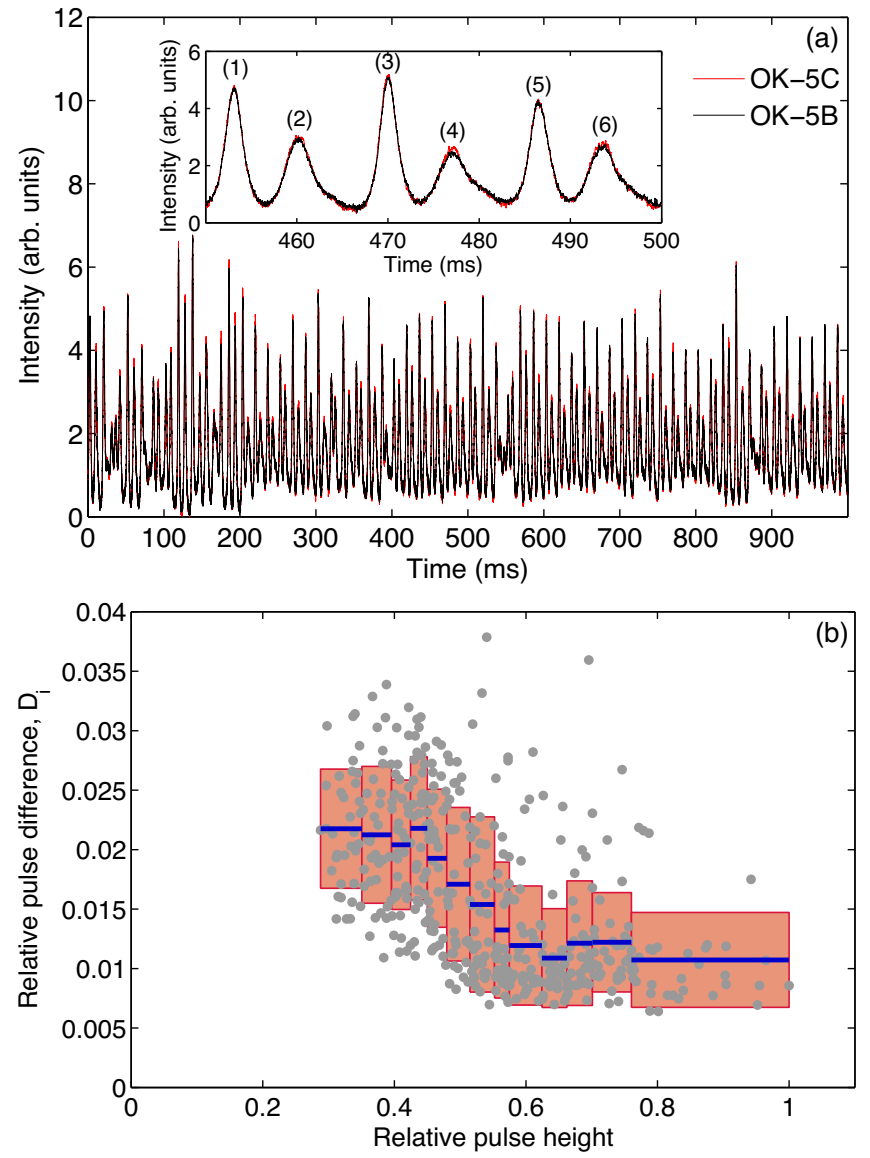

FIG. 5. FEL macropulses of the two orthogonal circular polarization components decomposed from an FEL beam with good average horizontal polarization. (a) FEL intensity measured in the time domain over one second. The inset is an enlarged plot, showing six consecutive FEL macropulses. (b) Relationship between the pulse difference $D_{i}$ and the pulse height for 455 macropulses collected from four one-second measurements (gray dots). The pulse height is normalized in a way to equalize the average intensity of the two macropulse signals. The data sorted into 13 bins using the pulse height are analyzed to show the averaged pulse difference (blue lines) and rms spread (red rectangles). For this measurement, the electron beam energy is $533 \mathrm{MeV}$ and beam current is $33 \mathrm{~mA}$ in the single bunch. The FEL is operated at $544 \mathrm{~nm}$ with close to zero FEL detuning.

transmission and sensitivity of the photodiodes and oscilloscope channels used, the two sets of data are normalized so that they have the same time-averaged intensity, reflecting the fact that the beam is close to perfect linear polarization in the dc measurement. The plotted data are smoothed using the moving average method with a $100 \mu$ s window and normalized to the total energy measured. As shown in this subplot, the pulses of two circular polarization components match very well for well-developed, tall pulses (e.g., pulses 1, 3, and 5 in the inset), but they show a slight mismatch around the peak for less-developed, lower pulses (e.g., pulses 2, 4, and 6 in the inset) and for the valley regions. Because the low-intensity regions have a small 
contribution to the time-averaged polarization measurements, the investigation focuses on the pulse mismatch around the peaks. To amplify these small discrepancies, we define for a particular pulse $i$ a dimensionless quantity to capture the pulse shape difference of the two circular polarization beam signals of opposite handedness:

$$
D_{i}=\frac{\int_{\Delta t}\left|I_{C}-I_{B}\right| d t}{\int_{\Delta t}\left(I_{C}+I_{B}\right) d t},
$$

where $I_{C}\left(I_{B}\right)$ is the measured intensity of the FEL beam associated with $\mathrm{OK}-5 \mathrm{C}(\mathrm{OK}-5 \mathrm{~B})$ undulator. The time integration is performed over a fixed time window $\Delta t$, which is selected to cover the peak symmetrically. Consequently, $D_{i}$ describes the relative pulse shape difference around the peak region. $\Delta t$ is chosen to be $2 \mathrm{~ms}$ to adequately capture the peak region of each pulse. $D_{i}$ is calculated for all reasonably developed pulses and plotted against the pulse height in Fig. 5(b), where data points are grouped into 13 bins in terms of the pulse height, with each bin containing 35 data points. In this subplot, each rectangle (in red) describes the statistics in a particular bin-the short blue line shows the averaged pulse difference, and the rectangle height shows the standard deviation $\left( \pm \sigma_{D}\right)$. This subplot shows that the pulse difference of two beam signals of opposite circular polarizations remains small $(1.1 \%-1.3 \%$, last six bins) and relatively stable for welldeveloped large pulses. However, when the pulse height is decreased to about one-half of the maximum height, the pulse difference starts to increase, by about a factor of 2 for the first seven bins as quantified using $D_{i}$. It is worth noting that the relative pulse difference remains at low levels, only $1.5 \%-2.2 \%$, even for those less-developed pulses.

Overall, the investigation of the FEL temporal structure reveals that all FEL macropulses in two orthogonal polarization states are generated with nearly perfect consistency in terms of the pulse's rise and fall times, duration, and height. This consistency is at the highest level for well-developed, narrower macropulses which are initiated when the electron beam has been better dampened with a smaller energy spread. In fact, there is no puzzle anymore: The observed nearly perfect temporal match of the intensity when the beam is separated into two different polarization states is the result of a narrow spectral linewidth of the FEL beam. The narrow spectral linewidth ensures that a set of cavity eigenmodes clustered around the modes with the highest gain will be amplified in a similar manner, while the wave packet reaches saturation with excellent linear polarization, for a given set of optimized operational parameters. This, in turn, ensures the unprecedentedly high degree of linear polarization as measured in terms of a time-averaged result using the dc method.

\section{SUMMARY}

Using two helical undulators with opposite helicities, we have experimentally demonstrated for the first time the generation of a fully controllable linearly polarized FEL beam using a storage ring FEL oscillator. This paper summarizes our strategies and techniques for producing an FEL beam with the unprecedented level of linear polarization: (i) exploring advantages of an FEL oscillator, (ii) configuring two mechanically identical helical undulators in the middle of the FEL cavity to operate with opposite helicities, (iii) developing a new FEL polarimeter which allows not only for a real-time optimization of the FEL beam polarization but also for highly accurate measurements of the degree of linear polarization, and (iv) tuning multiple operational parameters such as the undulator field strengths, buncher setting, FEL optical axis, and FEL detuning. By comparing the detuned and on-resonance FEL operations, we have demonstrated that the highest degree of linear polarization is the consequence of achieving a narrow spectral linewidth of the FEL beam. With all these efforts, we have produced highly polarized FEL beams $\left(P_{\text {lin }}>0.99\right)$ with a rotatable direction of linear polarization routinely for a range of operational parameters. In addition to dc measurements, the temporal structure of this FEL beam has been investigated-the pulsed beam intensity of the two circular polarization components associated with $\mathrm{OK}-5 \mathrm{~B}$ and $\mathrm{OK}-5 \mathrm{C}$ undulators, respectively, agree extremely well at the macropulse timescale with the welldeveloped macropulses showing the highest degree of temporal consistency. This finding provides new insight into how this FEL beam generated inside an oscillator possesses a very high degree of linear polarization as measured using the dc power measurements. With such a high degree of temporal consistency of the FEL macropulses, we also expect an excellent polarization consistency from "micropulse" to "micropulse." In fact, we are very interested in studying on a "micropulse-to-micropulse" basis the polarization buildup during the entire FEL gain process. While recognizing a power increase of about 6 orders of magnitude in the FEL lasing process makes such measurements challenging, this will be one of our future research topics.

This new optics-free polarization control method is well suited for high intracavity power operation of an FEL oscillator and, in principle, can be extended to the x-ray regime by using a future $x$-ray FEL oscillator [41,51,52]. At the HIGS, we have utilized this technique to produce $\gamma$-ray beams with switchable linear polarization. This has made the HIGS a more versatile $\gamma$-ray source with new polarization capabilities. This is critical for a number of important nuclear physics experiments by allowing the experimental setups to be simplified and systematic errors to be significantly reduced. This polarization control technique can also be used in the pulsed mode of the FEL operation, in which FEL macropulses are generated regularly using a gain modulation device. The pulsed, polarized gamma-ray beams are essential for certain experiments in which cosmic-ray background suppression is critical. Because 
of the wavelength limitation of this FEL polarimeter, $\gamma$-ray polarization control at the HIGS is currently available for low-energy $\gamma$-ray operation (1-30 MeV). To extend this capability to higher $\gamma$-ray energies, a new FEL polarimeter for the ultraviolet (UV) and vacuum ultraviolet (VUV) spectral regions is under development. With the use of fluoride-based optics, this system will be used to extend the polarization control capability of the Duke FEL to VUV wavelengths, which, in turn, will extend this polarization control technique to the higher HIGS operation energy, from 30 to $65 \mathrm{MeV}$ in the near future.

\section{ACKNOWLEDGMENTS}

This research was carried out at the High Intensity Gamma-ray Source facility at Triangle Universities Nuclear Laboratory (TUNL), a U.S. Department of Energy (DOE) Center of Excellence. We thank J.M. Mueller, M. W. Ahmed, and M. H. Sikora for developing a $\gamma$-ray polarimeter to experimentally confirm the high polarization quality of Compton $\gamma$-ray beams produced using this reported polarization controllable FEL. We are grateful to P. Wallace for helping improve the manuscript. We also acknowledge the support provided by the engineering and technical staff at Duke Free Electron Laser Laboratory/TUNL. This work is partially supported by DOE Grant No. DE-FG02-97ER41033.

[1] J. M. J. Madey, Stimulated emission of bremsstrahlung in a periodic magnetic field, J. Appl. Phys. 42, 1906 (1971).

[2] A. Renieri, Storage ring operation of the free-electron laser: The amplifier, Nuovo Cimento B 53, 160 (1979).

[3] R. Bonifacio, C. Pellegrini, and L. M. Narducci, Collective instabilities and high-gain regime in a free electron laser, Opt. Commun. 50, 373 (1984).

[4] G. R. Neil, C. Bohn, S. Benson, G. Biallas, D. Douglas, H. Dylla, R. Evans, J. Fugitt, A. Grippo, J. Gubeli et al., Sustained Kilowatt Lasing in a Free-Electron Laser with Same-Cell Energy Recovery, Phys. Rev. Lett. 84, 662 (2000).

[5] P. Emma, R. Akre, J. Arthur, R. Bionta, C. Bostedt, J. Bozek, A. Brachmann, P. Bucksbaum, R. Coffee, F.-J. Decker et al., First lasing and operation of an ångstrom-wavelength freeelectron laser, Nat. Photonics 4, 641 (2010).

[6] P. Neyman, W. B. Colson, S. C. Gottshalk, A. M. M. Todd, J. Blau, and K. Cohn, Free electron lasers in 2017, in Proceedings of the 38th International Free Electron Laser Conference (FEL'17), Santa Fe, NM, 2017 (JACoW, Geneva, 2018), pp. 204-209.

[7] D. Jaroszynski, R. Prazeres, F. Glotin, and J. Ortega, TwoColor Free-Electron Laser Operation, Phys. Rev. Lett. 72, 2387 (1994).

[8] G. Andonian, A. Murokh, J. B. Rosenzweig, R. Agustsson, M. Babzien, I. Ben-Zvi, P. Frigola, J. Y. Huang, L. Palumbo, C. Pellegrini et al., Observation of Anomalously
Large Spectral Bandwidth in a High-Gain Self-Amplified Spontaneous Emission Free-Electron Laser, Phys. Rev. Lett. 95, 054801 (2005).

[9] A. A. Lutman, R. Coffee, Y. Ding, Z. Huang, J. Krzywinski, T. Maxwell, M. Messerschmidt, and H.-D. Nuhn, Experimental Demonstration of Femtosecond TwoColor X-Ray Free-Electron Lasers, Phys. Rev. Lett. 110, 134801 (2013).

[10] A. Marinelli, A. A. Lutman, J. Wu, Y. Ding, J. Krzywinski, H.-D. Nuhn, Y. Feng, R. N. Coffee, and C. Pellegrini, Multicolor Operation and Spectral Control in a GainModulated X-Ray Free-Electron Laser, Phys. Rev. Lett. 111, 134801 (2013).

[11] E. Hemsing, A. Knyazik, M. Dunning, D. Xiang, A. Marinelli, C. Hast, and J. B. Rosenzweig, Coherent optical vortices from relativistic electron beams, Nat. Phys. 9, 549 (2013).

[12] Y. K. Wu, J. Yan, H. Hao, J. Y. Li, S. F. Mikhailov, V. G. Popov, N. A. Vinokurov, S. Huang, and J. Wu, Widely Tunable Two-Color Free-Electron Laser on a Storage Ring, Phys. Rev. Lett. 115, 184801 (2015).

[13] D. Gauthier, P. R. Ribič, G. De Ninno, E. Allaria, P. Cinquegrana, M. B. Danailov, A. Demidovich, E. Ferrari, L. Giannessi, B. Mahieu et al., Spectrotemporal Shaping of Seeded Free-Electron Laser Pulses, Phys. Rev. Lett. 115, 114801 (2015).

[14] A. Marinelli, R. Coffee, S. Vetter, P. Hering, G. N. West, S. Gilevich, A. A. Lutman, S. Li, T. Maxwell, J. Galayda et al., Optical Shaping of X-Ray Free-Electron Lasers, Phys. Rev. Lett. 116, 254801 (2016).

[15] D. Gauthier, P. R. Ribič, G. De Ninno, E. Allaria, P. Cinquegrana, M. B. Danailov, A. Demidovich, E. Ferrari, and L. Giannessi, Generation of Phase-Locked Pulses from a Seeded Free-Electron Laser, Phys. Rev. Lett. 116, 024801 (2016).

[16] E. Allaria, B. Diviacco, C. Callegari, P. Finetti, B. Mahieu, J. Viefhaus, M. Zangrando, G. De Ninno, G. Lambert, E. Ferrari et al., Control of the Polarization of a VacuumUltraviolet, High-Gain, Free-Electron Laser, Phys. Rev. X 4, 041040 (2014).

[17] H. Deng, T. Zhang, L. Feng, C. Feng, B. Liu, X. Wang, T. Lan, G. Wang, W. Zhang, X. Liu et al., Polarization switching demonstration using crossed-planar undulators in a seeded free-electron laser, Phys. Rev. Accel. Beams 17, 020704 (2014).

[18] E. Ferrari, E. Allaria, J. Buck, G. De Ninno, B. Diviacco, D. Gauthier, L. Giannessi, L. Glaser, Z. Huang, M. Ilchen et al., Single shot polarization characterization of XUV FEL pulses from crossed polarized undulators, Sci. Rep. 5, 13531 (2015).

[19] A. A. Lutman, J. P. MacArthur, M. Ilchen, A. O. Lindahl, J. Buck, R. N. Coffee, G. L. Dakovski, L. Dammann, Y. Ding, H. A. Dürr et al., Polarization control in an x-ray free-electron laser, Nat. Photonics 10, 468 (2016).

[20] J.F. De Boer and T. E. Milner, Review of polarization sensitive optical coherence tomography and Stokes vector determination, J. Biomed. Opt. 7, 359 (2002).

[21] J. F. De Boer, C. K. Hitzenberger, and Y. Yasuno, Polarization sensitive optical coherence tomography-A review, Biomed. Opt. Express 8, 1838 (2017). 
[22] B. M. Bulheller, A. Rodger, and J. D. Hirst, Circular and linear dichroism of proteins, Phys. Chem. Chem. Phys. 9, 2020 (2007).

[23] B. Ranjbar and P. Gill, Circular dichroism techniques: Biomolecular and nanostructural analyses-A review, Chem. Biol. Drug Des. 74, 101 (2009).

[24] G. van der Laan, Applications of soft x-ray magnetic dichroism, J. Phys. Conf. Ser. 430, 012127 (2013).

[25] G. van der Laan and A. I. Figueroa, X-ray magnetic circular dichroism-A versatile tool to study magnetism, Coord. Chem. Rev. 277, 95 (2014).

[26] S. D. Drell and A. C. Hearn, Exact Sum Rule for Nucleon Magnetic Moments, Phys. Rev. Lett. 16, 908 (1966).

[27] S. B. Gerasimov, A sum rule for magnetic moments and the damping of the nucleon magnetic moment in nuclei, Sov. J. Nucl. Phys. 2, 430 (1966).

[28] J. Ahrens et al., Helicity Dependence of $\gamma p \rightarrow N \pi$ below $450 \mathrm{MeV}$ and Contribution to the Gerasimov-Drell-Hearn Sum Rule, Phys. Rev. Lett. 84, 5950 (2000).

[29] J. Ahrens et al., First Measurement of the GerasimovDrell-Hearn Integral for ${ }^{1} \mathrm{H}$ from 200 to $800 \mathrm{MeV}$, Phys. Rev. Lett. 87, 022003 (2001).

[30] D. Drechsel, B. Pasquini, and M. Vanderhaeghen, Dispersion relations in real and virtual Compton scattering, Phys. Rep. 378, 99 (2003).

[31] M. Schumacher, Polarizability of the nucleon and Compton scattering, Prog. Part. Nucl. Phys. 55, 567 (2005).

[32] S. Sasaki, Analyses for a planar variably-polarizing undulator, Nucl. Instrum. Methods Phys. Res., Sect. A 347, 83 (1994).

[33] K. J. Kim, A synchrotron radiation source with arbitrarily adjustable elliptical polarization, Nucl. Instrum. Methods 219, 425 (1984).

[34] J. Bahrdt, A. Gaupp, W. Gudat, M. Mast, K. Molter, W. B. Peatman, M. Scheer, T. Schroeter, and C. Wang, Circularly polarized synchrotron radiation from the crossed undulator at BESSY, Rev. Sci. Instrum. 63, 339 (1992).

[35] K.-J. Kim, Circular polarization with crossed-planar undulators in high-gain FELs, Nucl. Instrum. Methods Phys. Res., Sect. A 445, 329 (2000).

[36] Y. K. Wu, N. A. Vinokurov, S. Mikhailov, J. Li, and V. Popov, High-Gain Lasing and Polarization Switch with a Distributed Optical-Klystron Free-Electron Laser, Phys. Rev. Lett. 96, 224801 (2006).

[37] Y. Ding and Z. Huang, Statistical analysis of crossed undulator for polarization control in a self-amplified spontaneous emission free electron laser, Phys. Rev. Accel. Beams 11, 030702 (2008).

[38] H. Geng, Y. Ding, and Z. Huang, Crossed undulator polarization control for X-ray FELs in the saturation regime, Nucl. Instrum. Methods Phys. Res., Sect. A 622, 276 (2010).
[39] Y. Li, B. Faatz, and J. Pflueger, Polarization properties of a crossed planar undulator, Nucl. Instrum. Methods Phys. Res., Sect. A 613, 163 (2010).

[40] S. Serkez, A. Trebushinin, M. Veremchuk, and G. Geloni, Method for polarization shaping at free-electron lasers, Phys. Rev. Accel. Beams 22, 110705 (2019).

[41] N. Huang, K. Li, and H. Deng, Polarization control of an $\mathrm{X}$-ray free electron laser oscillator, Phys. Rev. Accel. Beams 23, 030702 (2020).

[42] E. Ferrari, E. Roussel, J. Buck, C. Callegari, R. Cucini, G. De Ninno, B. Diviacco, D. Gauthier, L. Giannessi, L. Glaser et al., Free electron laser polarization control with interfering crossed polarized fields, Phys. Rev. Accel. Beams 22, 080701 (2019).

[43] J. Yan, J. M. Mueller, M. W. Ahmed, H. Hao, S. Huang, J. Li, V. N. Litvinenko, P. Liu, S. F. Mikhailov, V. G. Popov et al., Precision control of gamma-ray polarization using a crossed helical undulator free-electron laser, Nat. Photonics 13, 629 (2019).

[44] Y. K. Wu et al., Commissioning and operation of wiggler switchyard system for Duke FEL and HIGS, in Proceedings of the 4th International Particle Accelerator Conference, IPAC 2013, Shanghai, China, 2013 (JACoW, Shanghai, China, 2013), MOPEA078.

[45] V. N. Litvinenko, B. Burnham, M. Emamian, N. Hower, J. M. J. Madey, P. Morcombe, P. G. O'Shea, S. H. Park, R. Sachtschale, K. D. Straub et al., Gamma-Ray Production in a Storage Ring Free-Electron Laser, Phys. Rev. Lett. 78, 4569 (1997).

[46] H. R. Weller, M. W. Ahmed, H. Gao, W. Tornow, Y. K. Wu, M. Gai, and R. Miskimen, Research opportunities at the upgraded $\mathrm{HI} \gamma \mathrm{S}$ facility, Prog. Part. Nucl. Phys. 62, 257 (2009).

[47] N. A. Vinokurov and A. N. Skrinsky, An optical range generator-Ultra-relativistic electrons klystron, technical report, Budker Institute of Nuclear Physics, Novosibirsk, Report No. INP 77-59, 1977.

[48] D. Goldstein, Polarized Light, revised and expanded, Optical engineering (CRC Press, Boca Raton, 2003).

[49] M. Bass, E. W. V. Stryland, D. R. Williams, and W. L. Wolfe, Handbook of Optics Volume II Devices, Measurements, and Properties, 2nd ed. (McGraw-Hill, New York, 1995).

[50] J. Yan, H. Hao, J. Y. Li, S. F. Mikhailov, V. G. Popov, N. A. Vinokurov, S. Huang, J. Wu, S. Günster, and Y. K. Wu, Storage ring two-color free-electron laser, Phys. Rev. Accel. Beams 19, 070701 (2016).

[51] K.-J. Kim, Y. Shvyd'ko, and S. Reiche, A Proposal for an X-Ray Free-Electron Laser Oscillator with an EnergyRecovery Linac, Phys. Rev. Lett. 100, 244802 (2008).

[52] K.-J. Kim and Y. V. Shvyd'ko, Tunable optical cavity for an X-ray free-electron-laser oscillator, Phys. Rev. Accel. Beams 12, 030703 (2009). 